\title{
ESTUDIO COMPARATIVO DE LOS PUERTOS SAN ANTONIO Y BUENAVENTURA CON MIRAS A LA MEJORA DE LA COMPETITIVIDAD: CASO COLOMBIA
}

\author{
Sofía Martínez Mosquera \\ Escuela de Negocios, Gestión y Sostenibilidad \\ Programa de Especialización en Gestión Empresarial \\ Especialista en Gestión Empresarial \\ somartinez@poligran.edu.co \\ Diana Marcela López Gil \\ Escuela de Negocios, Gestión y Sostenibilidad \\ Programa de Especialización en Gestión Empresarial \\ Especialista en Gestión Empresarial \\ dilopezg2@poligran.edu.co \\ Daniela Alejandra Ojeda Triana \\ Escuela de Negocios, Gestión y Sostenibilidad \\ Programa de Especialización en Gestión Empresarial \\ Especialista en Gestión Empresarial \\ daojedat@poligran.edu.co
}

\section{Resumen}

El Puerto de Buenaventura representa el impacto más alto en la Economía Colombiana siendo su cobertura el $52 \%$ de la carga que se ingresa al país al año. Este tiene una ubicación estratégica, equidistante a la mayoría de las rutas importantes del mundo. Incrementado su capacidad de recepción, podría generarse una mayor competitividad logística. Recientemente, se inauguraron obras y equipos que aumentaron la capacidad del puerto para atender la demanda que representa la ampliación del Canal de Panamá, dicha inversión está enfocada en reducir los tiempos para generar un cargue y descargue más ágil, lo que permitirá revisar el costo de los fletes, tanto de los exportadores como los importadores. Colombia, se encuentra actualmente situada en una ubicación que le genera una ventaja para realizar comercialización a nuevos mercados como lo es el asiático. Por lo que realizaremos un análisis comparativo entre 2 puertos de gran impacto no solamente en Latinoamérica sino en el mundo, como lo son Buenaventura y San Antonio.

Palabras clave: logística portuaria; puerto; competitividad; infraestructura. 


\section{Introducción}

A continuación, se elaborará un estudio histórico y del estado del arte de los principales temas asociados al estudio comparativo.

\subsection{Metodología}

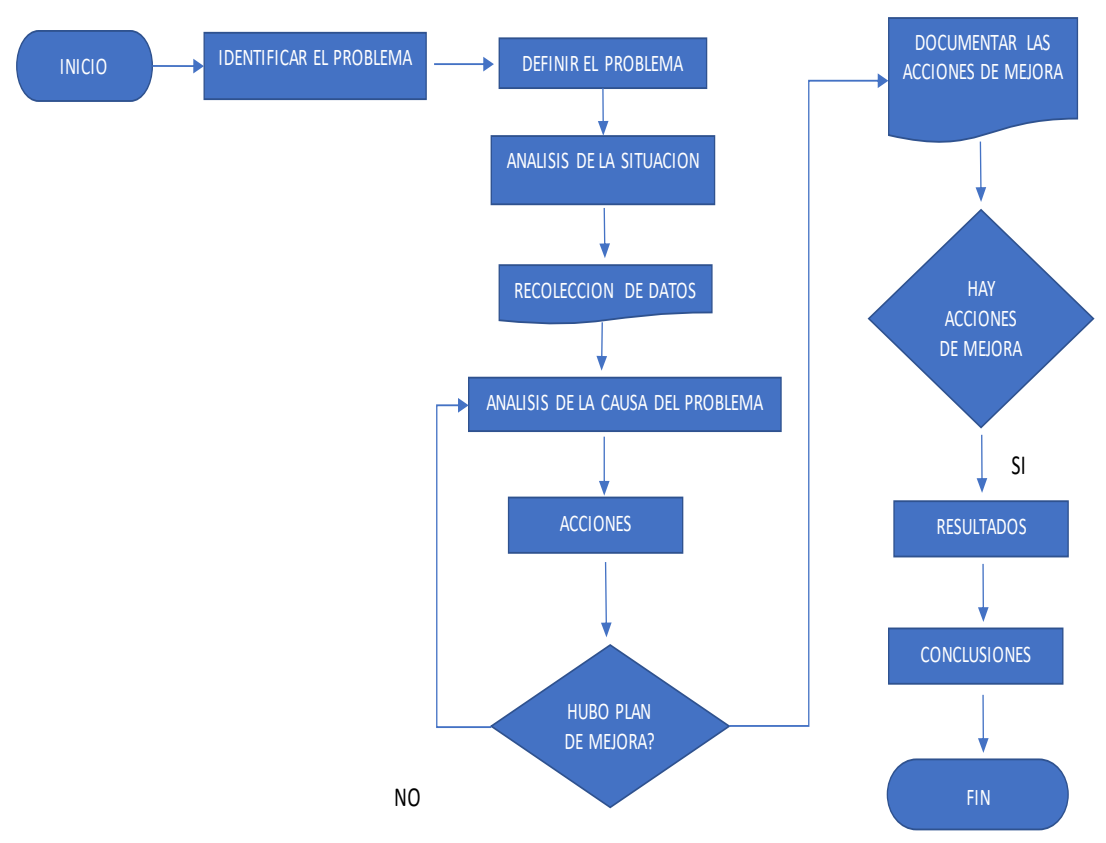

Imagen 1. Diagrama metodología

Recurso: propio

La imagen muestra el proceso metodológico que se siguió en la realización del presente artículo, partiendo de la identificación un área problemática asociada a la gestión del puerto de Buenaventura en Bogotá - Colombia. De considerar que la metodología tiene ciclos cerrados de retroalimentación lo cual permitió realizar una aproximación iterativa al problema y al análisis del mismo. Finalmente se aportan resultados y se plantean unas conclusiones asociadas al desarrollo de la investigación.

\subsection{El Puerto y su historia}

En 1925 el Puerto de Buenaventura movilizaba la quinta parte de la carga del país, aproximadamente el $15 \%$ de las exportaciones de café. Colombia ha tomado bastante ventaja frente a su crecimiento económico, por esto ha sido fuente de estudio debido a su geografía. Se encuentra ubicado en un sitio perfecto, donde es de fácil accesibilidad para los barcos de carga, así mismo, puede moverse a cualquier parte del mundo. 
La Costa Pacífica colombiana se encuentra separada del interior del país por cadenas de montañas, que se conocen como la cordillera occidental, así mismo predomina el clima cálido (por debajo de los $1.000 \mathrm{msnm}$. Buenaventura está situado en la parte izquierda de la cordillera occidental, limitando al norte con el Departamento del Chocó, al sur con Cauca y al Oriente con Cali.

El puerto de Buenaventura posee unas características especiales. Es el puerto con mayor participación en el comercio exterior, representando el 50\% de toda la carga nacional, que se puede ver en el volumen de importaciones (Perez, 2017).

\subsection{Puertos marítimos de Colombia}

En Colombia se presenta un alto porcentaje de transporte de carga marítima, esto se ha visto reflejado como una causa consecuente desde finales de los 80s, aproximadamente 37 millones de toneladas. Esto impulsado por el tratado de libre comercio con Estados Unidos, debido a esto todos los puertos ha venido desarrollando mejoras en su infraestructura, modernización de equipos y ampliación de la capacidad de movilización de carga. (PortalPortuario, 2018).

Los puertos de Colombia fueron manejados por el Estado, hasta el año de 1993, pero varios incidentes políticos llevaron a la liquidación de los puertos y en consecuencia fueron entregados a la Sociedad Portuarias Regionales (SPRs) quienes se enfocaron en mejorar las tarifas y su modernización.

Colombia cuenta con tres tipos de puertos:

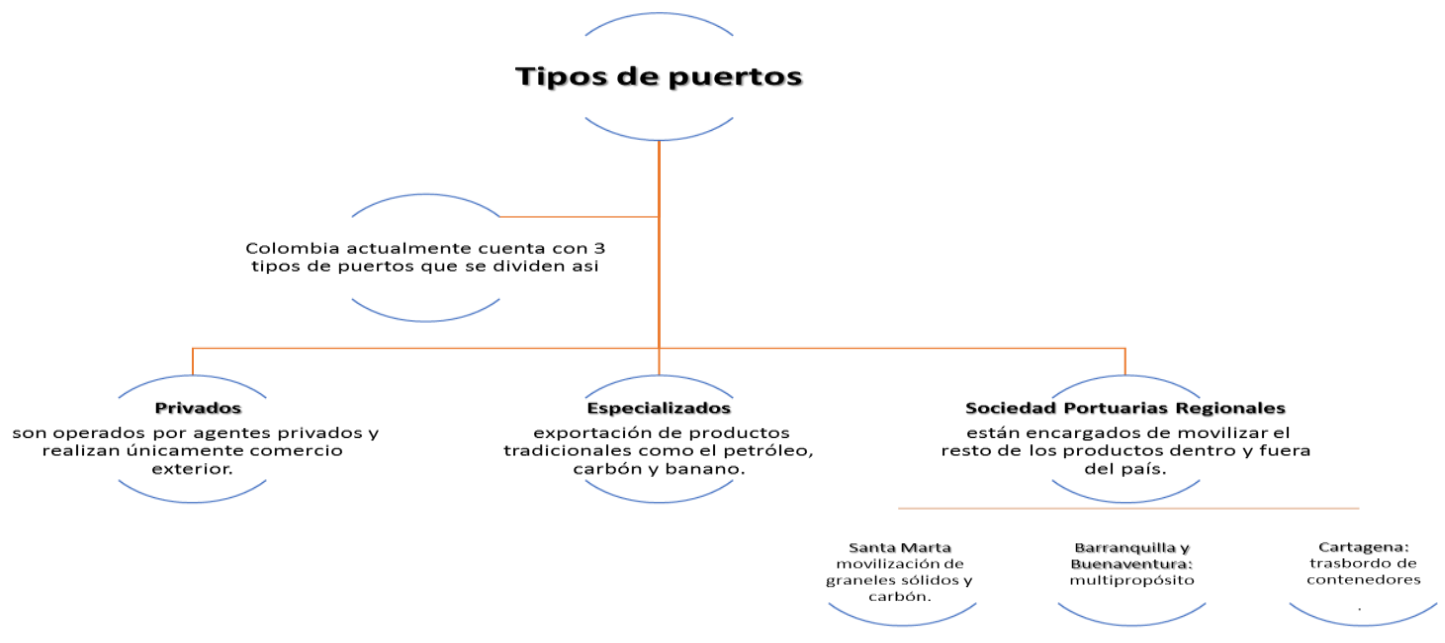

Imagen 2. Tipos de puertos

Recurso: propio 


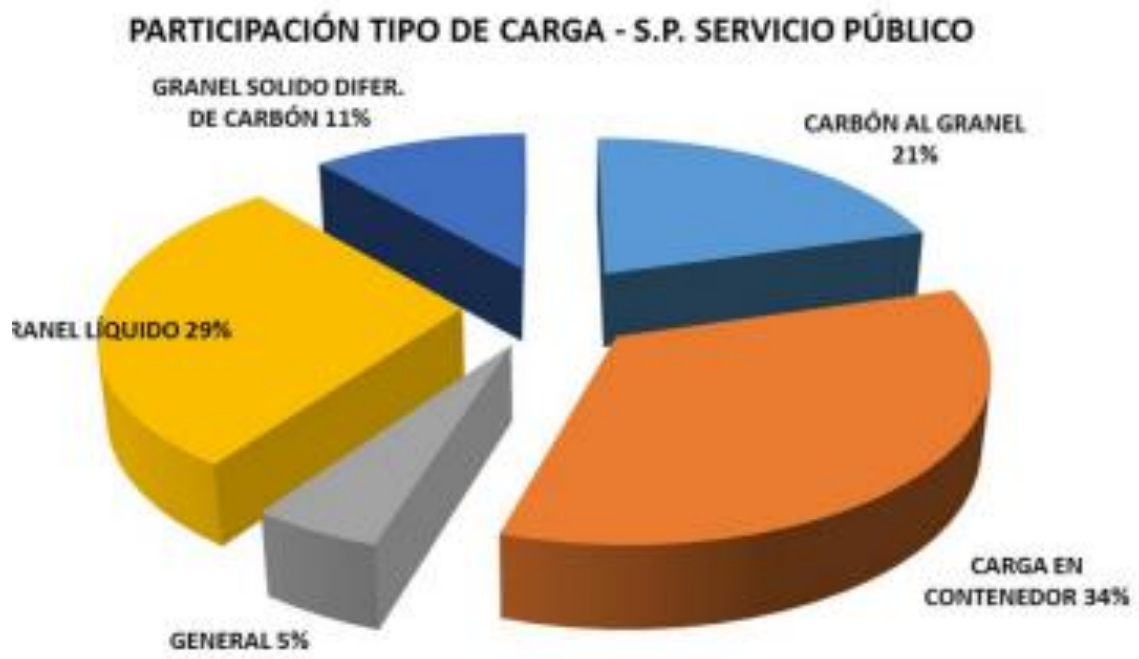

Imagen 3. Participación por tipo de carga

Recurso: www.supertransporte.gov.co (super tranporte, 2017)

Los puertos con mayor movilización son los regionales aproximadamente con un $65 \%$ del volumen total, le siguen los privados y su importancia se da debido a que mueven el petróleo, banano y carbón (Styles, 2018).

\subsection{Puertos secos}

Puertos que se encuentran ubicados al interior de las ciudades donde su mayor ventaja son sus vías, ya sean férreas, carreteras o conectadas a rutas marítimas si bien no son los más comunes actualmente han mostrado ser muy eficientes y con una gran capacidad de crecimiento y competitividad frente a puertos marítimos sin embargo la sostenibilidad en el largo plazo de estos puertos, se ve afectada por su ubicación geográfica ya que debe ser estratégicamente posicionado en las ciudades con el fin de permitir el acceso y la conexión con los puertos marítimos.

Los puertos más eficientes a nivel mundial están integrados en una cadena de cooperación mutua, en su gestión están involucradas empresas encargadas de las licencias para construcción de vías y de esta forma se genera un entendimiento de la dinámica de crecimiento de las zonas y su retorno de inversión. Esta situación impacta directamente la oferta de dichos puestos. Como resultado se genera una mejor gestión en las vías. El principal problema que enfrenta actualmente el puerto de buenaventura está en su logística exterior, ya que las vías destinadas a la evacuación de mercancía del puerto no son aptas para evacuar de manera eficiente el puerto y generan retrasos en el despacho de las mercancías hacia sus diferentes destinos (Ireta, 2010).

Se puede encontrar un ejemplo con la firma del acuerdo entre Astillero Río Santiago y Coserena, quienes firmaron un acuerdo de cooperación y complementariedad. 


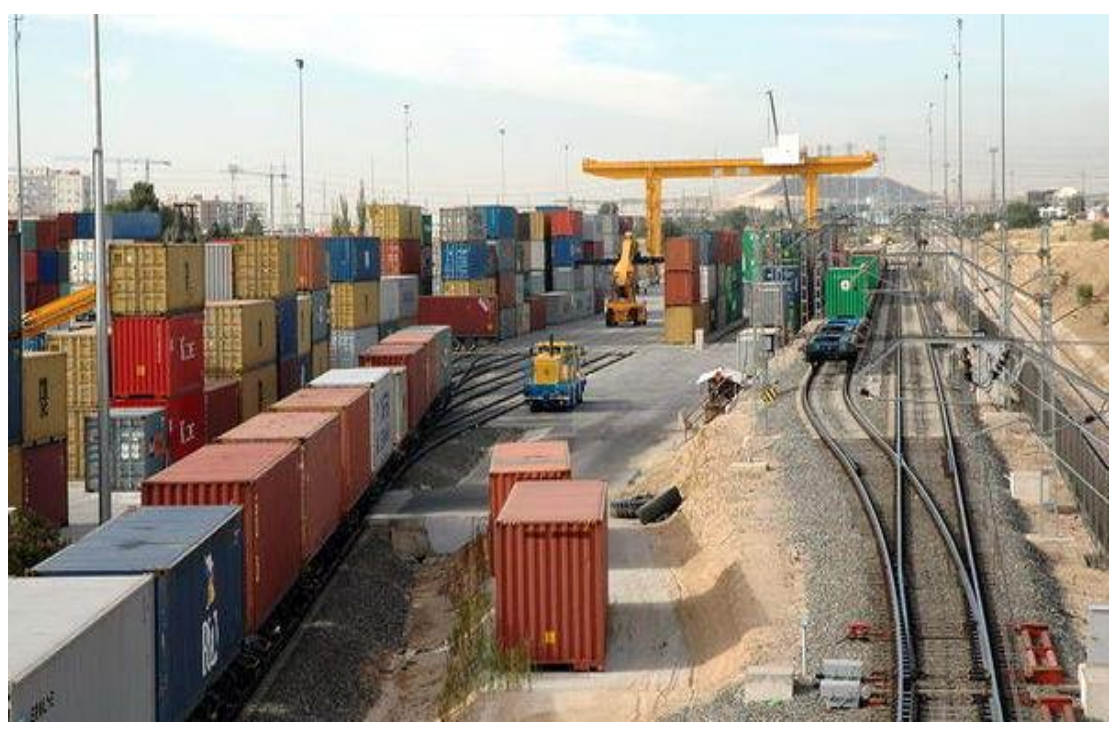

Imagen 4. Puerto seco

Recurso: www.noticiaslogisticaytransporte.com (noticiasylogisticatransporte, 2016)

El puerto de Buenaventura debe ser visto como el corazón del transporte de mercancía, y las vías de acceso constituyen sus arterias, por lo tanto, no importa que tan bueno, grande o innovador sea el corazón, si sus arterias no tienen la capacidad de movilizar la suficiente cantidad de mercancía tanto de entrada como de salida, eventualmente se generara un colapso y eso es lo que hoy por hoy constituye un factor crítico para el puerto de Buenaventura.

\subsection{Ventaja competitiva en materia de movilidad}

Uno de los aspectos a considerar teniendo en cuenta lo mencionado en el punto anterior es que estos puertos secos representan una ventaja competitiva directamente asociada a sus rutas, y comparativamente es el factor crítico dominante para el puerto de buenaventura cabe analizar que si se evidencia y trabaja en este factor crítico relacionado con las vías del puerto de buenaventura este quizás podría ser uno de los puertos más eficientes, sino el más eficiente de Latinoamérica.

Si bien en la actualidad Colombia solo cuenta con puertos marítimos, está sobre la mesa la "terminal de la 80" un puerto seco que sería un centro de distribución hacia Bogotá que evitaría que los grandes vehículos entraran a Bogotá lo que tendría beneficios en 3 aspectos, movilidad, malla vial y descontaminación, lo que permitiría una mayor fluidez en la movilización de TEUS a lo largo del territorio nacional. Por la conexión que este tendría incluso directamente con el aeropuerto.

\subsection{El Puerto de Buenaventura}


El Puerto de Buenaventura cuenta actualmente con 12 muelles y es compartido por la Armada Nacional y la Sociedad Portuaria Regional de Colombia. Su ubicación se puede ver en la imagen 5.

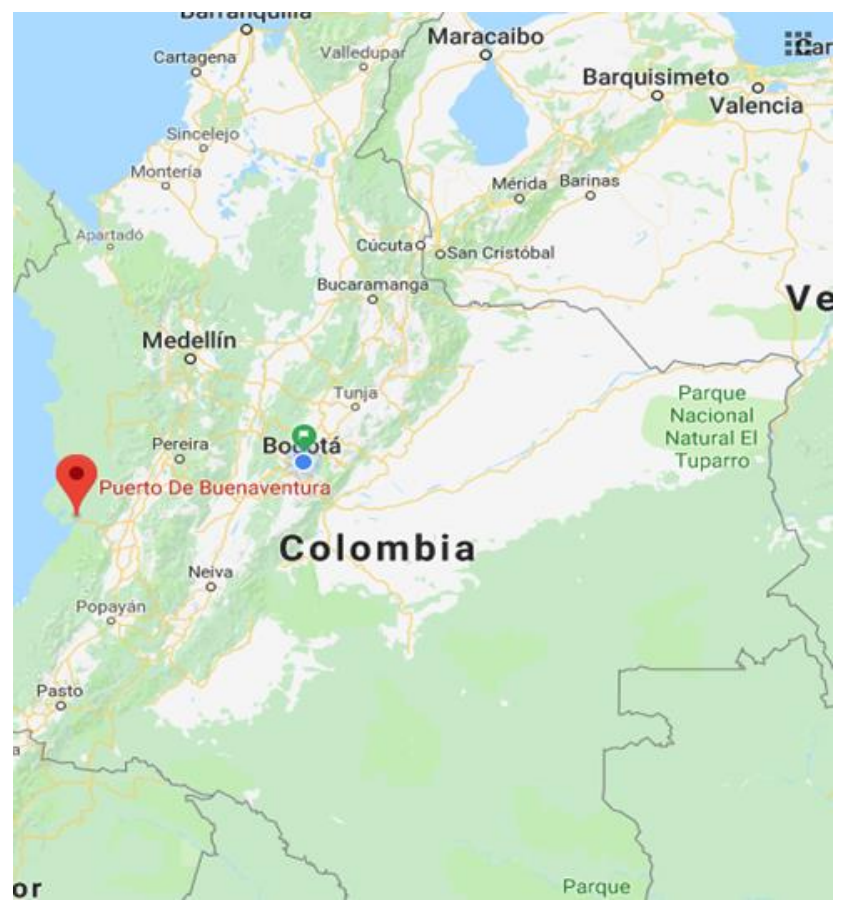

Imagen 5. Ubicación geográfica

Recurso: Google Maps

\subsection{Concesiones Actuales}

El gobierno ha entregado 4 concesiones que tienen como objetivo la mejora del puerto cuya finalidad es lograr que se convierta en el más innovador. Es importante tener en cuenta que la empresa La Sprbun tiene el 70\% de estas concesiones; es una empresa de capital mixto de la cual el $83 \%$ corresponde al sector privado y solo el $15 \%$ es de la alcaldía de buenaventura y un $2 \%$ que le corresponde al ministerio de transporte.

Visto desde esta óptica es realmente fácil entender porque no se ven reflejadas las inyecciones de capital hasta la ciudad de buenaventura, pues solo el $2 \%$ está asignado a el ministerio de transporte, y si bien, este es uno de los problemas más grandes con los que cuenta el puerto hasta ahora, sus rutas de acceso dejan mucho que desear, la demora en la construcción de los túneles de la línea son un indicio de lo grave que es este problema pues estas básicamente se convierten en un embudo para el puerto. 


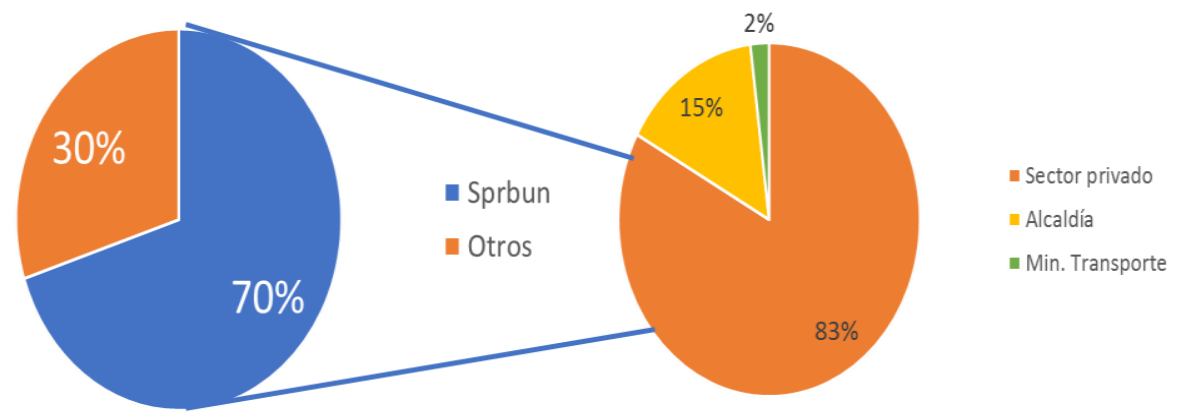

Gráfica 1. Porcentaje de concesiones

Recurso: propio

Basado en los datos, se puede evidenciar que es el reflejo de una desventaja muy grande frente a países como chile, así se quiera ser el país más innovador, puerto con mayor capacidad y logística moderna, si las vías de acceso no están adecuadas para aumentar el rendimiento y la eficiencia de la logística portuaria, no será atractivo. A pesar de las alianzas y tratados de libre comercio que se tienen en el país para incrementar el porcentaje de importaciones y exportaciones, la competitividad se ve afectada por el alto costo en los fletes. Por eso es importante que estas concesiones de cientos de millones de dólares se extiendan también hacia las vías. 


\begin{tabular}{|c|c|c|c|c|c|c|c|c|}
\hline \multirow{3}{*}{ Zona Portuaria } & \multicolumn{6}{|c|}{ Tercer Trimestre } & \multirow{3}{*}{$\begin{array}{c}\text { Variación } \\
\% \\
2015-2016\end{array}$} & \multirow{3}{*}{$\begin{array}{c}\text { Variación } \\
\% \\
\text { 2016-2017 }\end{array}$} \\
\hline & \multicolumn{2}{|c|}{2015} & \multicolumn{2}{|c|}{2016} & \multicolumn{2}{|c|}{2017} & & \\
\hline & $\begin{array}{c}\text { Nro. } \\
\text { Arribos }\end{array}$ & $\begin{array}{l}\text { Part. } \\
(\%)\end{array}$ & $\begin{array}{c}\text { Nro. } \\
\text { Arribos }\end{array}$ & $\begin{array}{l}\text { Part. } \\
(\%)\end{array}$ & $\begin{array}{c}\text { Nro. } \\
\text { Arribos }\end{array}$ & $\begin{array}{l}\text { Part. } \\
(\%)\end{array}$ & & \\
\hline Z.P. Región Caribe & 6,291 & 77.9 & 6,486 & 78.2 & 6,790 & 81.3 & 3.1 & 4.7 \\
\hline Cartagena & 3,416 & 54.3 & 3,581 & 56.9 & 3,402 & 54.1 & 4.8 & -5.0 \\
\hline Barranquilla & 1,070 & 17.0 & 1,078 & 17.1 & 972 & 15.5 & 0.7 & -9.8 \\
\hline Santa Marta & 787 & 12.5 & 857 & 13.6 & 933 & 14.8 & 8.9 & 8.9 \\
\hline San Andrés & 290 & 4.6 & 234 & 3.7 & 233 & 3.7 & -19.3 & -0.4 \\
\hline Guajira & 363 & 5.8 & 330 & 5.2 & 320 & 5.1 & -9.1 & -3.0 \\
\hline Cienaga & 365 & 5.8 & 406 & 6.5 & 401 & 6.4 & 11.2 & -1.2 \\
\hline Golfo Morrosquillo & 390 & 6.2 & 355 & 5.6 & 347 & 5.5 & -9.0 & -2.3 \\
\hline Turbo & - & - & - & - & 182 & 2.9 & 0.0 & 0.0 \\
\hline Z.P. Región Pacifica & 1,244 & 15.4 & 1,019 & 12.6 & 902 & 11.2 & -18.1 & -11.5 \\
\hline Buenaventura & 1,212 & 97.4 & 999 & 80.3 & 880 & 70.7 & -17.6 & -11.9 \\
\hline Tumaco & 32 & 2.6 & 20 & 1.6 & 22 & 1.8 & -37.5 & 10.0 \\
\hline Z.P. Río Magdalena & 540 & 6.7 & 788 & 9.5 & 661 & $\mathbf{0}$ & 45.9 & -16.1 \\
\hline Barrancabermeja & 347 & 64.3 & 643 & 119.1 & 570 & 105.6 & 85.3 & -11.4 \\
\hline Rio Magdalena & 193 & 35.7 & 145 & 26.9 & 91 & 16.9 & -24.9 & -37.2 \\
\hline Total Arribos & 8,075 & 100 & 8,293 & 100 & 8,353 & 100 & 2.7 & 0.7 \\
\hline
\end{tabular}

Imagen 6. Transporte en puertos de Colombia Recurso: Recurso: www.supertransporte.gov.co (Transporte en puertos de Colombia, 2017)

Unidad: Número de arribos 
La imagen 6, representa el volumen en el 2015, 2016 y primer semestre del 2017, donde se puede notar un factor constante en el que el Puerto de Buenaventura representa un alto porcentaje comparado con el resto del país.

La mayor cantidad de arribos se hacen al puerto de Cartagena, cuya representación total dentro de las zonas portuarias totales del país corresponde al $54 \%$, mientras que esta representación por parte del puerto de buenaventura se ubica en el 4 lugar con un total de 880 arribos para el 2017.

\subsection{El Puerto de San Antonio - Chile}

Actualmente, se presenta una necesidad que se exige por el mercado; fácil acceso y mayor seguridad en los servicios que se están prestando, solo de esta forma se podrá aumentar la competitividad.

En Colombia, según la Encuesta Nacional logística del 2015, los costos logísticos respecto al total de las ventas representan el 14,97\%, donde el transporte (representa el $37 \%$ en el valor del costo) y el almacenamiento (representa el $20 \%$ en el valor del costo). Se debe tener en cuenta que los costos logísticos son los más representativos para el valor final del producto (Gaviria S. , 2015).

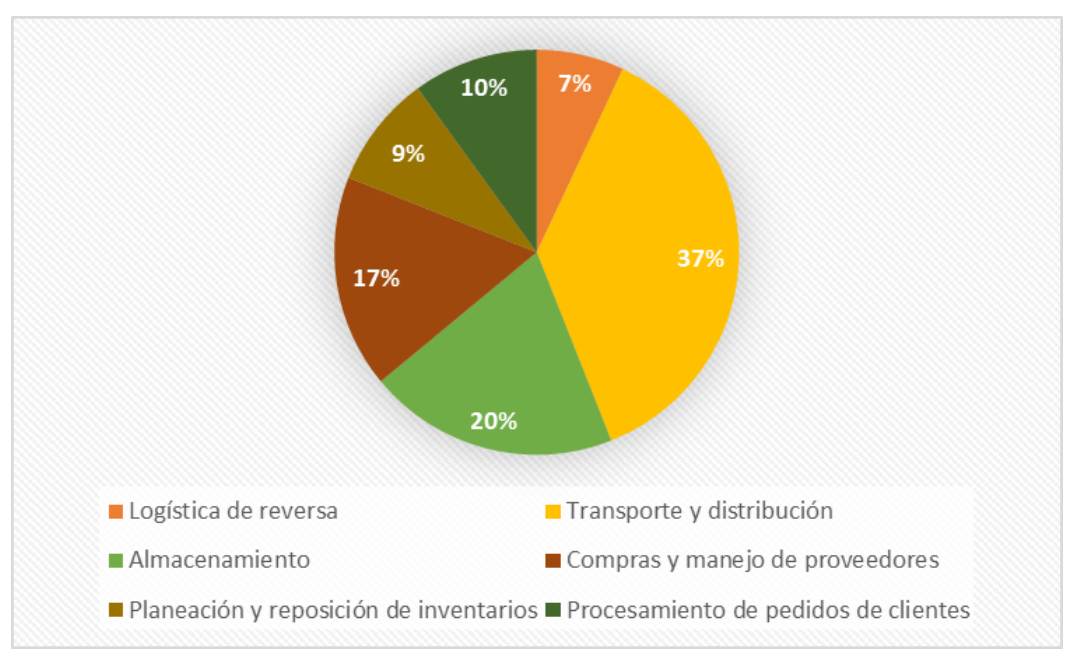

Grafica 2. costos logísticos respecto a la participación el total de las ventas Recurso: https://onl.dnp.gov.co/.../Encuesta Nacional Logística 22015

En relación con el desempeño calculado por el Banco Mundial en el 2014, las cifras no favorecen a Colombia para aumentar su competitividad (Muñoz, Encuesta\%20Nacional\%20Logística\%202015\%20-\%20, 2015). 


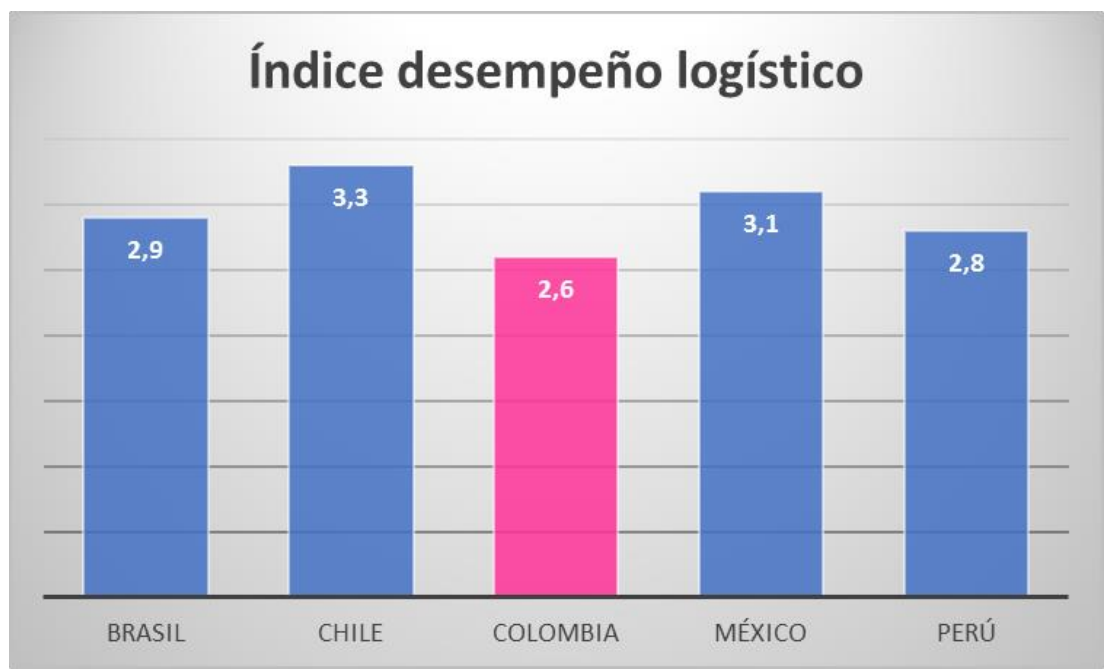

Grafica 3. Índice de Desempeño Logístico

Recurso: https://onl.dnp.gov.co/Encuesta Nacional Logística 22015

En cuanto a la logística portuaria de uno de los más influyentes de Latinoamérica es el Puerto de San Antonio en Chile, y uno de los factores que se resaltan para que su participación a nivel portuario sea una de las más importantes, es la colaboración de una cadena de actores que son grandes responsables para el impacto y determinación del posicionamiento del desarrollo del país.

En primer lugar, como actor principal son los habitantes que residen en la ciudad, para el caso de San Antonio se genera el 50\% de empleabilidad en el movimiento portuario, comercio turismo y pesca lo cual es una ventaja competitiva dado que este grupo de personas es generador para el apoyo de proyectos y crecimiento del puerto, igualmente la participación de ellos es muy valiosa para escuchar sus inquietudes a todo lo que respecta frente al puerto.

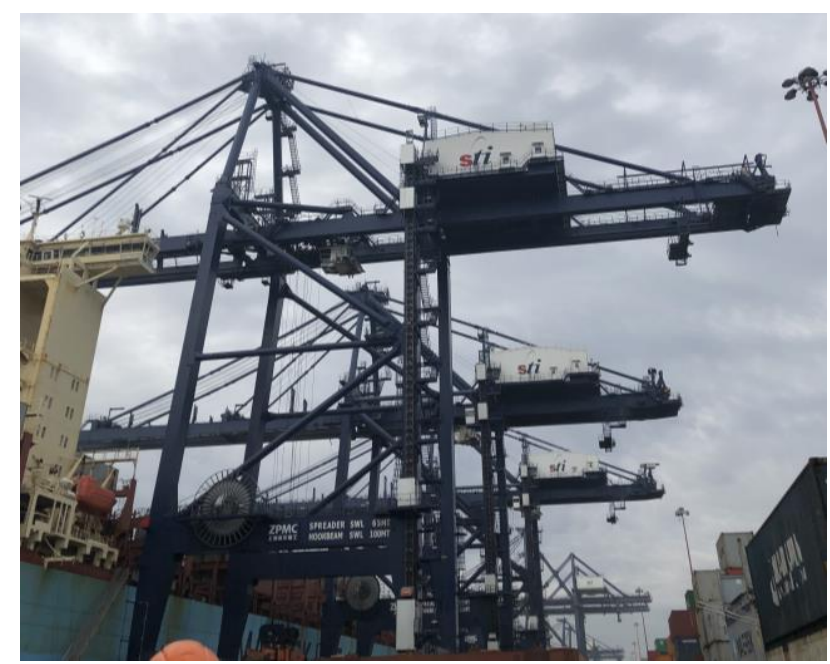

Imagen 7. Puerto de San Antonio (chile)

Recurso: propio 
En segundo lugar, como otro actor principal que ha influido al crecimiento de este puerto son las concesiones que reúnen todos los organismos a nivel de empresas privadas, instituciones gubernamentales y organismos en donde todos trabajan para un mismo fin: la competitividad, y el desarrollo sustentable del puerto. Estas concesiones ayudaron al mejoramiento de la administración de los puertos de Chile (Echeverría1, 2012).

\subsubsection{Estrategias de crecimiento}

Desde el momento que el gobierno ve una oportunidad de crecimiento económico en gran medida por el comercio exterior y las ventajas competitivas que tiene por ser un país costero, empiezan a crear estrategias que puedan llevar a cabo la idea de poner a Chile entre los países más importantes en el tema de logística Portuaria en Latinoamérica, de esta manera es resaltar algunas de las estrategias que fueron determinantes para el posicionamiento que se encuentra actualmente (CARRILLO, nov. 2005).

Algunas de la toma de decisiones fue la intervención de la inversión privada donde algunos de los objetivos fueron: descentralización portuaria, mejoramiento en la infraestructura de los puertos y calidad del servicio. Con estas estrategias lo que se pretendía era la implementación en el mejoramiento de tecnología, igualmente reducir tarifas y costos portuarios de tal manera que disminuyera los tiempos de espera en naves y el ahorro de fletes debido a la recepción de naves de mayor tamaño, por último, todo esto determinaba una calidad en el servicio y mejor atención.

Como resultado de la implementación de estas estrategias en los últimos años Chile ha incrementado en transferencias de carga siendo más competitivos en el tema tarifario y disminución de horas en Latinoamérica.

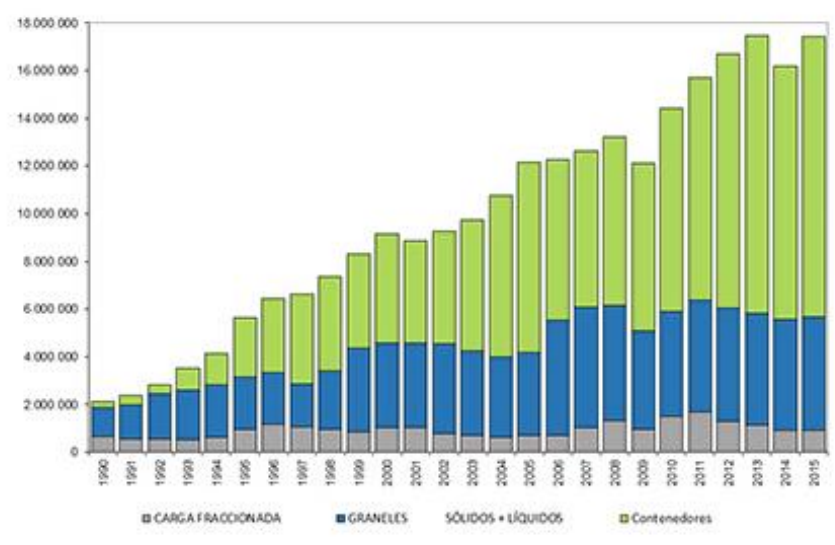

Imagen 8. Evolución Histórica Anual

Tipo de Carga Puerto San Antonio

Recurso: http://www.sanantonioport.cc.cl/html/estadisticas/mensual.php (Evolución Mensual por tipos de Carga) 


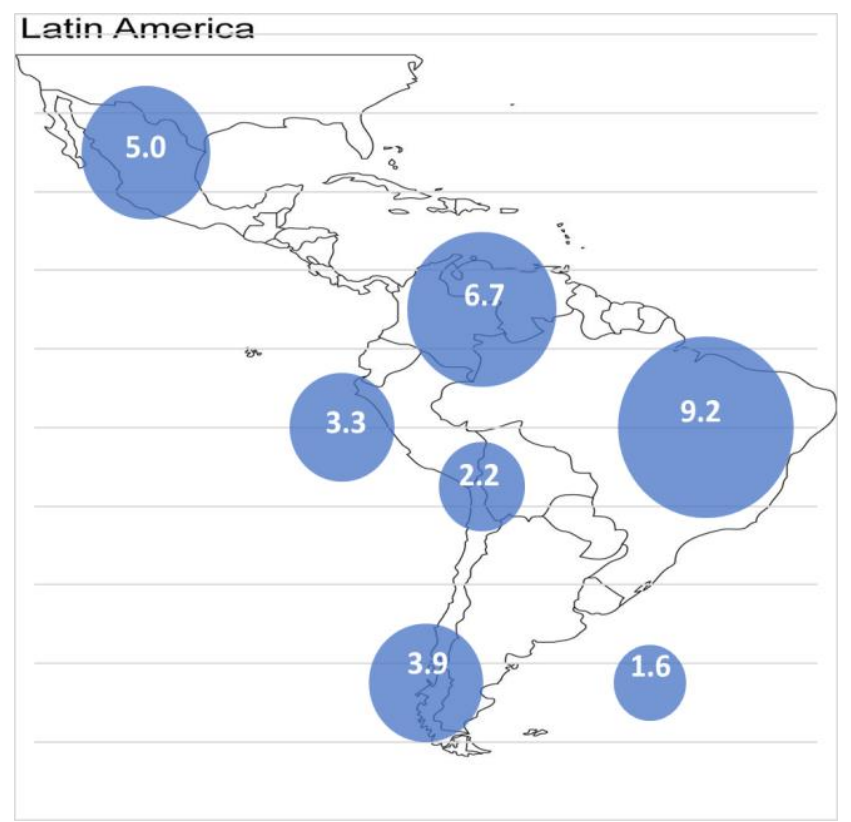

Gráfica 4. Rankin de puertos según CEPAL (Comisión Económica para América Latina y el Caribe) en el 2014

Recurso: www.cepal.org Elaboración: propio

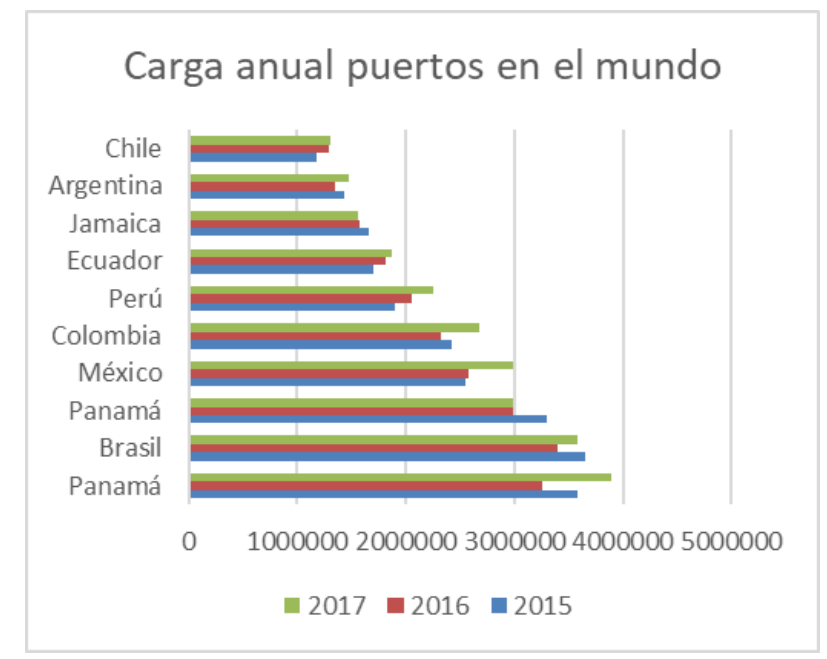

Gráfica 5. Rankin de puertos según CEPAL (Comisión Económica para América Latina y el Caribe) en el 2015,2016 y 2017

Recurso: propio

En la gráfica 5, se puede observar el crecimiento que ha tenido el puerto de Chile en su movimiento de carga del 2015 al 2017. 
Para poderse considerar como un país competitivo en el tema de distribución y comercialización de mercancías, hay que tener en cuenta unos factores claves para el desarrollo y sostenibilidad, de esta manera el puerto de San Antonio en Chile tiene estos factores con lo cual lo tiene entre los mejores puertos de Latinoamérica.

\begin{tabular}{|c|c|c|}
\hline FACTORES & PUERTO BUENAVENTURA / COLOMBIA & PUERTO SAN ANTONIO/CHILE \\
\hline $\begin{array}{l}\text { Ubicación } \\
\text { geográfica: }\end{array}$ & $\begin{array}{l}\text { *Ubicación estratégica dada su proximidad con las } \\
\text { principales rutas de comercio internacional, permité } \\
\text { un aumento progresivo del transporte de } \\
\text { mercancías }\end{array}$ & $\begin{array}{l}\text { *Ubicado en la zona Central, de influencia conformada por } \\
\text { la macrozona centro-sur del país y el Mercosur. }\end{array}$ \\
\hline $\begin{array}{l}\text { Infraestructura } \\
\text { adecuada: }\end{array}$ & $\begin{array}{l}\text { *Altos costos de transporte } \\
\text { * Insuficientes carreteras, puertos y aeropuertos }\end{array}$ & $\begin{array}{l}\text { * La infraestructura vial chilena está compuesta por una red } \\
\text { de carreteras de } 80.505 \mathrm{~km} \text {. que se extiende por todo el } \\
\text { país, de las cuales } 16.745 \mathrm{~km} \text { están pavimentadas. Chile } \\
\text { posee una infraestructura portuaria compuesta por más de } \\
24 \text { puertos que están habilitados para el manejo de } \\
\text { diferentes tipos de carga, lo que permite que el } 95 \% \text { del } \\
\text { comercio exterior del país se transporte por este medio. }\end{array}$ \\
\hline $\begin{array}{l}\text { Desarrollo de } \\
\text { comunicaciones: }\end{array}$ & $\begin{array}{l}\text { *Carencia en el uso de tecnología para tener } \\
\text { trazabilidad de la carga desde origen hasta destino. } \\
\text { *Falta de sistemas de información en logística. }\end{array}$ & $\begin{array}{l}\text { * Capacidad para procesar información relacionada con el } \\
\text { comercio y trasporte y de tal forma que sea más rápida y } \\
\text { segura, tales como las plataformas, las bases de datos con } \\
\text { las que se cuenta, medios electrónicos, son claves para } \\
\text { aumentar la rapidez en operaciones y despacho de carga } \\
\text { favoreciendo la integración de la cadena logística. }\end{array}$ \\
\hline Calidad Servicio & $\begin{array}{l}\text { *Incumplimiento en los tiempos de entrega. } \\
\text { *Alto valor de los fletes frente a la calidad del } \\
\text { servicio ofrecido. }\end{array}$ & $\begin{array}{l}\text { * Tarifas razonables, competitivas y con un aumento de las } \\
\text { velocidades de transferencia de la carga y disminución de } \\
\text { las horas de espera por falta de sitiodisminución de los } \\
\text { costos y los tiempos de la cadena de transporte en su } \\
\text { conjunto. }\end{array}$ \\
\hline
\end{tabular}

Gráfica 6. Factores comparativos entre puerto de buenaventura y San Antonio Recurso: propio

\section{Puerto de Buenaventura un reto para la economía.}

Buenaventura un puerto con alta proyección para impulsar las importaciones y exportaciones en el país. ¿Qué retos se tienen?, ¿Qué se puede mejorar?

\subsection{Diagnóstico actual}

Se realizará el diagnóstico actual del Puerto de Buenaventura, basados en los estudios y comparaciones del Puerto de San Antonio en Chile, que representa altos porcentajes de importación y exportación. El análisis del puerto representará diferentes variables que nos pintarán un mapa más detallado de la logística, infraestructura y administración del puerto, los cuales serán abordados para aumentar las capacidades y fomentar un servicio de alta competitividad. (Portafolio, 2018)

Se identifican y comparan indicadores, con base a estos se plantean mejoras en el puerto de buenaventura. El objetivo será identificar los inconvenientes en la logística portuaria para proponer planes de mejora que representen ahorros, generación de ingresos e inversión al país 
aumentando la capacidad logística, mejorando la infraestructura del puerto y sus vías acceso. En relación con la clasificación de los puertos se destaca unos grupos que van a ser objeto de estudio en este artículo que se consideran los más importantes:

Como un primer grupo se compone por los servicios de abastecimiento y logística. El manejo adecuado de la cadena de suministro es importante para un buen desarrollo en el sector logístico.

Como segundo grupo corresponde a la calidad y el nivel de desarrollo de accesibilidad al interior de las actividades portuarias, con este grupo se quiere destacar que una infraestructura de alta calidad permite la accesibilidad fácil de los mercados del interior y el estar bien posicionados para mejorar y reforzar sus posiciones competitivas internacionales de servicio

Por último, como tercer grupo se refiere al comercio, que corresponden a todos los actores que participan para una buena comunicación en el puerto: apoyo financiero, grupos de la industria, sectores comerciales etc.

\subsection{Distancia y recorrido (Chile)}

En la imagen 10. se evidencia que hay una línea rosa, indica la distancia que existe entre Santiago, y el Puerto San Antonio esta asciende a $89.62 \mathrm{~km}$, mientras que la línea azul, refleja el recorrido que tendría que hacerse por carretera que sería de $104.82 \mathrm{~km}$, el tiempo estimado para este recorrido es de 1 hora y 4 minutos. Lo que se traduce en una velocidad promedio de $80 \mathrm{~km}$ por hora.

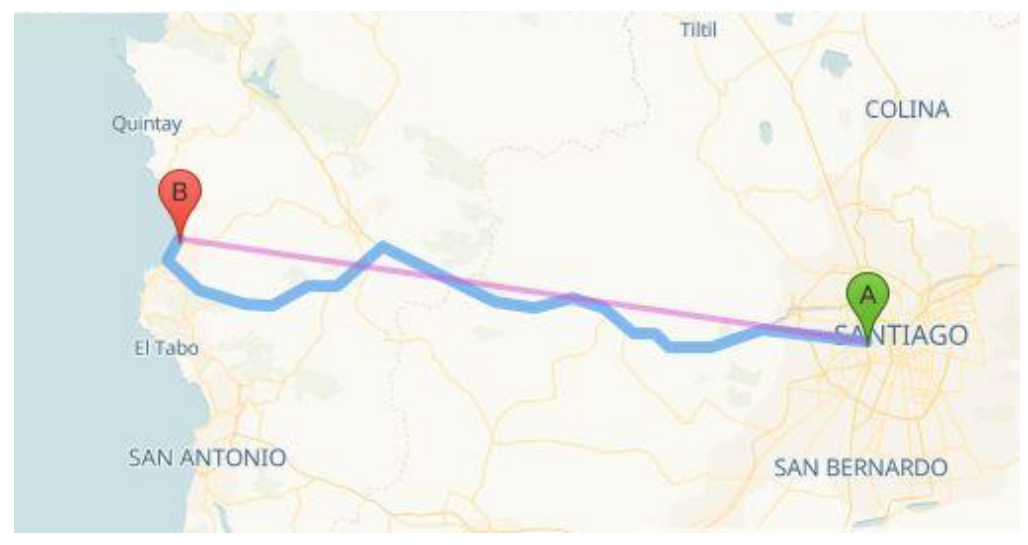

Imagen 10. Recorrido y distancia entre Santiago y el puerto de San Antonio Recurso: www.distanciaentrecuidades.com (cálculo distancias)

Esta corta distancia ligada de las excelentes carreteras con las que cuenta chile, representa una ventaja competitiva no solamente para el puerto sino para el país ya que el tiempo que tardan en transportar un contenedor es relativamente corta.

\subsection{Distancia y recorrido (Colombia)}


En la imagen 11. La línea rosa muestra una distancia de $339.55 \mathrm{~km}$ entre Bogotá y Buenaventura, mientras que la línea azul que es la que indica las carreteras muestra un recorrido de $528.29 \mathrm{~km}$, recorrer esta distancia toma un total de 7 horas y $2 \mathrm{~min}$. Aunque tomando información del transporte de un contenedor es de 11 horas y 11 minutos por lo que nos arroja una velocidad promedio de $48 \mathrm{~km}$.

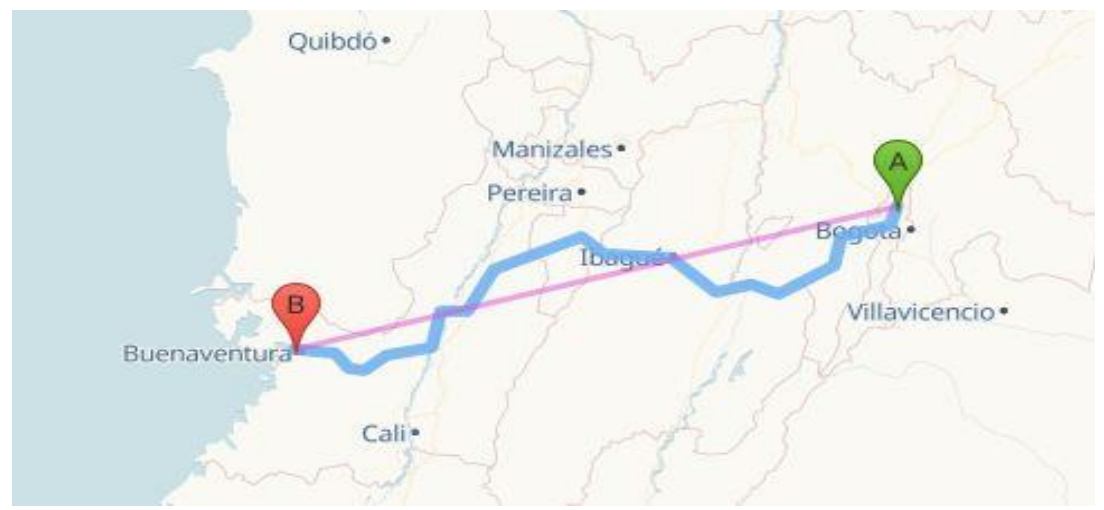

Imagen 11. Recorrido y distancia entre Bogotá y el puerto de Buenaventura Recurso: www.distanciaentrecuidades.com (cálculo distancias)

Esto a modo comparativo ya demarca una desventaja para el puerto de buenaventura porque no solamente es un recorrido que marca casi 1/3 parte de un día, sino que adicionalmente las carreteras en Colombia no están en condiciones óptimas lo cual impide que este recorrido se realice de manera continua y efectiva.

\subsection{Puerto de Buenaventura Vs. Puerto de San Antonio}

A manera comparativa podemos evidenciar la movilización de TEUS en el puerto de san Antonio Vs. el de Buenaventura entre 2014 al 2015 el puerto de San Antonio movilizó en promedio 248 mil TEUS, más que el puerto de Buenaventura, pero para el 2016 esta cifra se duplicó llegando a unos $422 \mathrm{mil}$.

El puerto de San Antonio presentó un incremento en la cantidad movilizada de 10,4\% mientras que en Buenaventura esta cifra cayó de 911.533 a 864.749 lo que se estima en una disminución del 5,13\% para los años 2015 y 2016. Mientras que el puerto de San Antonio muestra un crecimiento constante, el puerto de buenaventura muestra una disminución en el año 2016 respecto al 2015 de 46 mil TEUS. 


\begin{tabular}{|r|l|l|r|r|r|r|}
\hline RANKING & PUERTO/PORT & PAIS/COUNTRY & 2014 (TEUS) & 2015 (TEUS) & 2016 (TEUS) & $\begin{array}{c}\text { VARIACION/ } \\
\text { CHANGE } \\
\% 2016 / 2015\end{array}$ \\
\hline 1 & Santos & Brasil & 3.569 .870 & 3.645 .448 & 3.393 .593 & $-6,9 \%$ \\
\hline 2 & Colon & Panamá & 3.286 .736 & 3.577 .427 & 3.258 .381 & $-8,9 \%$ \\
\hline 3 & Balboa & Panamá & 3.468 .283 & 3.294 .113 & 3.989 .860 & $21,1 \%$ \\
\hline 4 & Manzanillo & México & 2.355 .149 & 2.541 .140 & 2.580 .660 & $1,6 \%$ \\
\hline 5 & Cartagena & Colombia & 2.239 .214 & 2.397 .969 & 2.301 .099 & $-4,0 \%$ \\
\hline 6 & Callao & Perú & 1.992 .473 & 1.900 .444 & 2.054 .970 & $8,1 \%$ \\
\hline 7 & Guayaquil & Ecuador & 1.621 .381 & 1.704 .730 & 1.821 .654 & $6,9 \%$ \\
\hline 8 & Kingston & Jamaica & 1.638 .113 & 1.653 .272 & 1.567 .442 & $-5,2 \%$ \\
\hline 9 & Buenos Aires & Argentina & 1.428 .843 & 1.433 .053 & 1.352 .368 & $-5,6 \%$ \\
\hline 10 & San Antonio & Chile & 1.093 .625 & 1.170 .184 & 1.287 .658 & $10,0 \%$ \\
\hline 11 & Freeport & Bahamas & 1.400 .000 & 1.400 .000 & 1.200 .000 & $-14,3 \%$ \\
\hline 12 & Limon-Moin & Costa Rica & 1.089 .518 & 1.108 .573 & 1.177 .385 & $6,2 \%$ \\
\hline 13 & Lazaro Cardenas & México & 996.954 & 1.058 .747 & 1.115 .452 & $5,4 \%$ \\
\hline 14 & Veracruz & México & 847.370 & 931.812 & 965.294 & $3,6 \%$ \\
\hline 15 & Caucedo & R.Dominicana & 831.375 & 826.935 & 918.542 & $11,1 \%$ \\
\hline 16 & Navegantes & Brasil & 676.675 & 662.590 & 895.375 & $35,1 \%$ \\
\hline 17 & Montevideo & Uruguay & 776.558 & 811.297 & 888.119 & $9,5 \%$ \\
\hline 18 & Valparaiso & Chile & 1.010 .202 & 902.542 & 884.030 & $-2,1 \%$ \\
\hline 19 & Buenaventura & Colombia & 855.404 & 911.533 & 864.749 & $-5,1 \%$ \\
\hline 20 & Paranaguá & Brasil & 757.319 & 782.346 & 725.041 & $-7,3 \%$ \\
\hline 19 & & & & \\
\hline
\end{tabular}

Imagen 9. Movimiento de contenedores principales puertos de Latinoamérica y el caribe (TEUS) (Movimiento de contenedores principales puertos de latinoamerica y el caribe (TEUS), 2017)

Recurso: $\underline{\text { www.supertransporte.gov.co }}$ 


\begin{tabular}{|c|c|c|}
\hline & Puerto de Buenaventura & Puerto San Antonio \\
\hline Localización & $\begin{array}{l}\text { Ubicado en el Pacífico, cerca al } \\
\text { canal de Panamá, equidistante } \\
\text { entre Vancouver y Valparaíso }\end{array}$ & $\begin{array}{l}\text { Ubicado en la zona central, siendo el } \\
\text { terminal portuario más cerca a Santiago. }\end{array}$ \\
\hline \multirow[t]{2}{*}{ Infraestructura } & $\begin{array}{l}\text { Vía marítima: canal de acceso de } \\
30 \text { km de longitud. }\end{array}$ & \multirow{2}{*}{$\begin{array}{l}\text { Cuatro frentes de ataque: molo sur, } \\
\text { espigón, terminal norte y policarpo toro } \\
\text { Suman un total de } 1399 \mathrm{~m} \text { de longitud. }\end{array}$} \\
\hline & $\begin{array}{l}\text { Muelles: } 177 \text { ha, } 13 \text { atraques y } \\
\text { longitud del muelle de } 1951 \text { m. }\end{array}$ & \\
\hline Almacenamiento & $\begin{array}{l}\text { Tres muelles especializados en } \\
\text { contenedores, pantalla de } 463 \\
\text { m y retaguardia de } 124.000 \mathrm{~m} 2 . \\
\text { Patio de almacenamiento de } \\
\text { 250.000 TEUs y capacidad de } \\
\text { 5.356 TEUs. }\end{array}$ & $\begin{array}{l}\text { Cuatro bodegas de } 11.000 \mathrm{~m} 2 \text {, superficie } \\
\text { de } 1,1 \text { hectáreas totalmente cerrada y } \\
\text { terminal de contenedores de } 7000 \mathrm{~m} 2 \text {. }\end{array}$ \\
\hline Superestructura & $\begin{array}{l}\text { Dos grúas pórtico, una grúa } \\
\text { móvil y } 6 \text { grúas pórtico. }\end{array}$ & $\begin{array}{l}4 \text { grúas porta contenedores del tipo } \\
\text { Granty, grúas tipo RTG, } 6 \text { equipos Rech } \\
\text { Stacker llenos y } 2 \text { equipos vacíos. } 12 \\
\text { tractocamiones y } 10 \text { unidades de chasis. } \\
2 \text { grúas Demag, } 1 \text { grúa Gottwald y } 20 \\
\text { equipos de porteo. }\end{array}$ \\
\hline Instalaciones complementarias & $\begin{array}{l}\text { No hay zona para la expansión, } \\
\text { ya que se encuentra cerca al } \\
\text { casco urbano. }\end{array}$ & \\
\hline
\end{tabular}

Gráfica 7. Cuadro comparativo puertos

Recurso: diagramación propia (Chapapria, 2003)

\section{Resultados del estudio}

El Puerto de Buenaventura se encuentra situado dentro de los que representan mayor movimiento en Latinoamérica. Se presentan déficits representativos en el ámbito de la infraestructura, las vías de acceso no son adecuadas para el movimiento de carga, por esto es uno de los puntos donde mayor foco se debe tener. (PortalPortuario, 2018). 
"Enviar un contenedor desde Cartagena a Bogotá cuesta tres veces más que enviarlo desde ese puerto de Colombia a Shangái” (Cosoy, 2015).

El costo por Km de llevar la carga de Buenaventura a Bogotá es de US\$3,58, mientras que en las grandes ciudades mover una carga oscila entre los US\$1,20 (Luis, 2015).

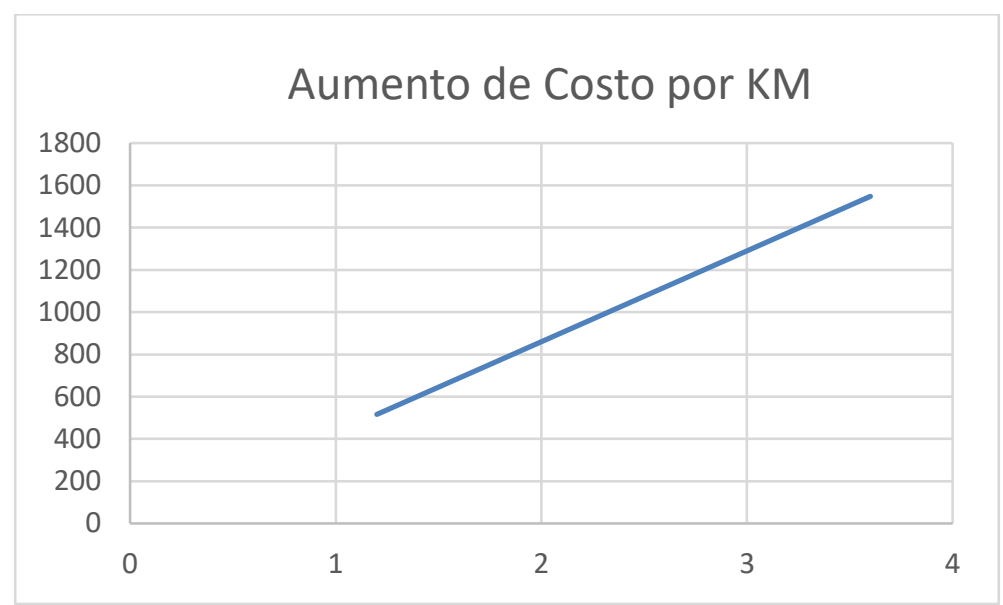

Gráfica 8. Aumento costo por KM - Buenaventura - Bogotá Recurso: propio

El costo es una función lineal, es decir, que si mejoramos la infraestructura de acceso al puerto disminuimos los costos de transporte y por ende, los costos del puerto.

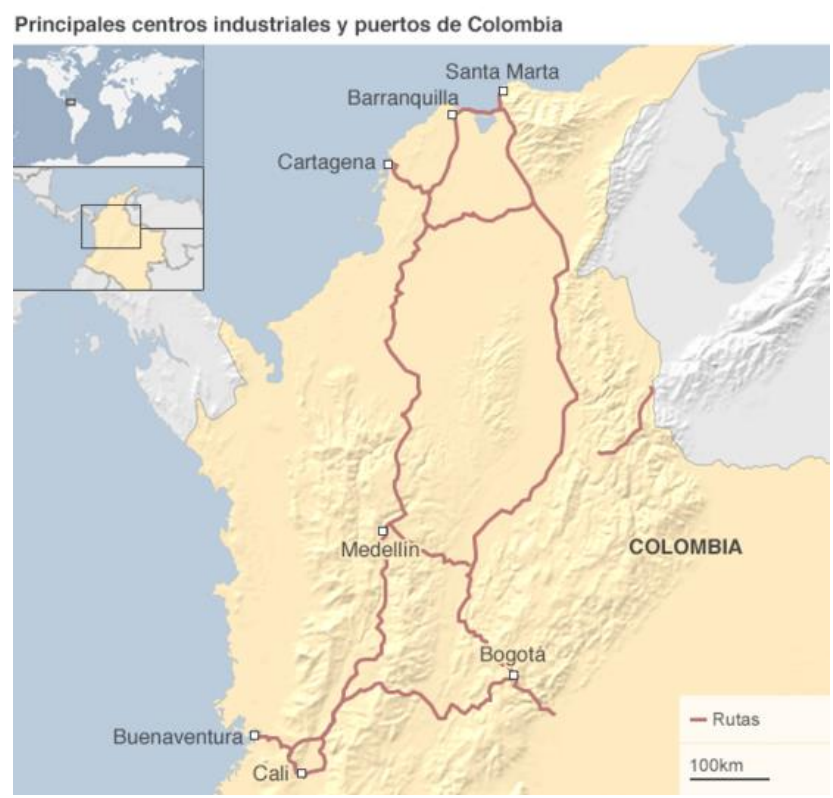

Imagen 12. Vías de acceso a los principales puertos

Recurso: BBC mundo 
Se propone un análisis económico donde realizar un km de vía en Colombia tiene un costo aproximado de 708 millones de pesos.

Se deben explorar soluciones que beneficien la infraestructura de conexión al puerto, pero también tengo un periodo de retorno corto. Teniendo en cuenta el costo de 708 millones de pesos, recuperaríamos aproximadamente en 74 años la inversión. Esto sin sumar, los costos que se ahorran, ya que un conductor no gastaría entre 9 y 10 horas en el trayecto de los 430 kilómetros, sino la mitad. (Díez, 2016).

Es decir que se debe contar con un periodo de retorno más extenso para poder rehabilitar los 430 kilómetros hasta la capital. Por esto, se proponen soluciones más rentables, como lo es la Ecovía o la estabilización con cemento. "La solución aplicada en el proyecto es un desarrollo exclusivo de la empresa que tiene por nombre EcoVía CEMEX.

Esta tecnología consiste en aprovechar el material existente en el suelo que, al ser mezclado con cemento y aditivos de CEMEX, mejora su capacidad de soporte. Esto, representa ventajas técnicas que se reflejan en el ahorro de transporte de material para la obra, consumo de combustible, y emisiones de CO2". Esto podrá representar un ahorro de aproximadamente el $50 \%$ en el costo de la construcción de la vía. Debe resaltarse que es un gran porcentaje de vías en conexión al puerto son terciarias (cemex colombia , 2017).

El tiempo se reduce a la mitad, así mismo el costo para el transportador y el empleador. Esto podrá representar una mayor ventaja para el movimiento logístico del puerto, mayor cantidad de transportistas movilizando cargas, en un menor tiempo y a un menor costo; el panorama ideal.

\section{Conclusiones.}

Como resultado de la investigación podemos concluir que existen retos importantes que se recomienda trabajar en el Puerto de Buenaventura, el cual en el trascurso del artículo se ha venido tratando. Una vez analizada la información que fue estudiada, se pueden concluir 3 aspectos que son importantes considerar en la transformación del puerto:

- Infraestructura interna.

- Infraestructura externa.

- Participación de la comunidad.

De estos 3 aspectos evidenciamos que, a nivel de infraestructura interna, actualmente se están haciendo grandes inversiones lo que le permitirá al puerto una ampliación en su capacidad de carga y así poder atender la demanda mundial en mayor capacidad de transporte de UTES.

Infraestructura externa, como se explicó a lo largo del documento es de gran importancia invertir en las carreteras, ya que se está generando un embotellamiento en la salida y entrada de los contenedores, lo que hace que el puerto pierda su eficiencia, ya que aumenta los tiempos 
de recepción y distribución de estos. Así mismo se propone la implementación de puertos secos que permitan una mejor evacuación de las cargas, lo que mejoraría no solo la movilidad portuaria sino la de la capital, conectando este tipo de puertos por vías férreas, permitiría la descongestión en el movimiento de cargas. No solo al interior de las ciudades sino también fuera de ellas.

La participación de la comunidad dentro del puerto es algo que se recomienda adaptar del puerto de San Antonio permitiendo la generación de inversión económica dentro de la ciudad por parte de los empleados y como consecuencia un sentido de pertenencia que le permitirá al puerto un crecimiento potencial y desarrollo de proyectos que lleven a la creación de una mayor capacidad competitiva. Tener aliados que trabajen y vivan en la zona no solo es importante para la generación de empleo, sino también para el crecimiento de Buenaventura.

Buenaventura tiene un potencial de crecimiento que podrá explotar y potencializar, si se enfoca en aumentar el rendimiento de su logística portuaria, es decir, mejorando las vías podrá disminuir el costo de los fletes y aumentar su proporción de envíos de cargas. De esta forma, se volverá atractivo para sus clientes, donde podrán enviar mayor a carga a un menor costo.

Incluyendo a su comunidad, permitirá que sientan que es de ellos, que deben cuidarlo y ayudar a impulsar su estabilidad. No solo impulsa el puerto, sino a la economía de Buenaventura, brindándole mayores posibilidades de empleo. 


\section{Bibliografía}

cálculo distancias. (n.d.). Retrieved from www.distanciaentrecuidades.com

CARRILLO. (nov. 2005). MODERNIZACIÓN PORTUARIA EN CHILE. Sínt. tecnol. [online]., 63-68.

cemex colombia . (2017). cemex . Retrieved from Invertimos 200 Millones De Pesos En El Mejoramiento Vías Terciarias De Jerusalén, Cundinamarca :

www.cemexcolombia.com

Chapapria, V. (2003, Junio). ADB. Retrieved from IADB:

www20.iadb.org/intal/catalogo/pe/2008/01721.pdf

Cosoy, N. (2015). BBC MUNDO. Retrieved from Por qué es tres veces más barato mandar un contenedor de Colombia a China que dentro de Colombia:

WWW.BBCMUNDO.COM

Díez, A. (2016, Mayo 2). Universidad de los Andes. Retrieved from Universidad de los Andes: https://agronegocios.uniandes.edu.co/2016/05/02/balance-de-nuestrainfraestructura/

Echeverría1, O. S. (2012). Análisis de la conectividad externa de los puertos de Chile como un factor de competitividad . Ingeniare. Revista chilena de ingeniería, vol. $20 \mathrm{~N}^{\mathrm{o}} 1$, 2012, pp. 25-39 .

Evolución Mensual por tipos de Carga. (n.d.). Retrieved from http://www.sanantonioport.cc.cl/html/estadisticas/mensual.php

Gaviria, S. (2015). ONL. Retrieved from https://onl.dnp.gov.co/.../Encuesta Nacional Logística 22015

Gaviria, S. (2015). ONL. Retrieved from https://onl.dnp.gov.co/.../Encuesta Nacional Logística 22015

Ireta, E. (2010, Noviembre). OAS. Retrieved from "Los Puertos y Su Conectividad." : www.oas.org/cip/docs/documentos_importantes/

Luis, F. (2015). BBC LATINOAMERICA. Retrieved from Los países con las mejores y las peores carreteras en A. Latina: www.bbcmundo.com

Movimiento de contenedores principales puertos de latinoamerica y el caribe (TEUS). (2017). Retrieved from www.supertransporte.gov.co

Muñoz, S. G. (2015). dpn. Retrieved from https://colaboracion.dnp.gov.co/CDT/Prensa/Publicaciones/Encuesta\%20Nacional\%2 0de\%20Log\%C3\%ADstica\%20Resultados\%202015\%20(002).pdf

Muñoz, S. G. (2015). Encuesta\%20Nacional\%20Logística\%202015\%20-\%20. Retrieved from https://onl.dnp.gov.co/... 
noticiasylogisticatransporte. (2016). noticiasylogisticatransporte. Retrieved from WWW.noticiaslogisticaytransporte.com

Perez, G. J. (2017, 04). Banco de la Republica. Retrieved from Banco de la Republica: http://www.banrep.gov.co/docum/Lectura_finanzas/pdf/DTSER-91.pdf

Portafolio. (2018, Mayo 06). Portafolio. Retrieved from Portafolio: http://www.portafolio.co/economia/infraestructura/los-avances-en-materia-portuariaque-ha-tenido-el-pais-516841

PortalPortuario. (2018, Abril 16). PortalPortuario. Retrieved from PortalPortuario: https://portalportuario.cl/colombia-sociedad-portuaria-de-buenaventura-pide-cambioa-contrato-de-concesion/

SANTANDER, I. C. (2005). MODERNIZACIÓN PORTUARIA EN CHILE. I. Carrillo. Síntesis Tecnológica, 12.

Styles, L. (2018, Abril 20). ”Digital Logistics Capacity Assessments. Retrieved from Colombia Puerto de Buenaventura;:

dlca.logcluster.org/display/public/DLCA/2.1.1Colombia Puerto de Buenaventura; jsessionid

super tranporte. (2017). Retrieved from www.supertransporte.gov.co

Transporte en puertos de Colombia. (2017). supertransporte. Retrieved from

www.supertranposrte.gov.co 